\title{
LA NOCIÓN DE ATRIBUTO EN LA LINGÜÍSTICA ESPAÑOLA
}

\author{
INMACULADA PENADÉS MARTÍNEZ \\ (Universidad de Cádiz)
}

0 . El concepto de atributo o de predicado nominal, términos estos más frecuentemente utilizados para designar uno de los componentes de las oraciones atributivas (los otros elementos son el sujeto y el verbo copulativo), suele ser estudiado por la mayor parte de los gramáticos a partir de las nociones de atribución y de verbo copulativo; sin embargo, aquí vamos a ocuparnos de él sin referirnos, previa y explícitamente, a las mismas.

1. Definición del atributo. En tres grupos podrían encuadrarse las distintas definiciones que hemos recogido $y$, así, al primer grupo pertenecerían las de la Academia, la de S. Gili Gaya y la de R. Seco ${ }^{1}$, que viẹnen a coincidir en su visión del concepto, pues, en los tres casos, se considera que el predicado nominal atribuye cualidades al sujeto, con lo que se le califica o clasifica mediante un adjetivo, un sustantivo o una frase o expresión con el sentido de éstos. Algo distintas son, en cambio, las definiciones de R. Navas, F. Marcos y J. Alcina y J. M. Blecua ${ }^{2}$, dado que en ellas se tiene en cuenta el verbo de la oración atributiva. En efecto, para el primero de estos autores, el atributo es el nombre, o lo que haga las veces de tal, que posee un valor adjetivo, que debe estar necesariamente en la frase atributiva y cuya función es expresar la manera de aparecer el sujeto o el complemento directo, teniendo en cuenta que éstos son definidos por el atributo, el cual depende de ellos mediante un verbo que lo introduce; por su parte, F. Marcos señala que el atributo significa una sustancia semántica predicativa que se une a la significación del su-

1 Véase, al respecto, R.A.E. (1931), pp. 154-156; R.A.E. (1977), p. 364; S. Gili Gaya (1976), p. 57, y R. Seco, (1971), pp. 131-132.

2 Véase, al respecto, R. Navas (1963), p. 34; F. Marcos Marín (1975), pp. 230-231, y J. Alcina y J. M. Blecua (1975), pp. 858-859. 
jeto mediante un verbo, $y$, por último, J. Alcina y J. M. Blecua definen el predicado nominal como un nombre unido al sujeto por un verbo, por lo que a través de éste la cualidad se atribuye a aquél. Otras veces, y ahora estaríamos en el tercer grupo, los gramáticos, al definir el atributo, insistirán en la importancia léxica del mismo, frente al carácter pretendidamente gramatical de los verbos copulativos. Asi, por ejemplo, J. Falk, entre otras características, apunta que el complemento predicativo, término con el que también se designa el atributo, contiene la información léxica del predicado. De manera hasta cierto punto análoga, C. Hernández afirma que la función semántica del atributo es completar la significación de los predicados ser y estar, precisando, delimitando y determinando su contenido verbal. Por su parte, E. Alarcos considera que el atributo es el término adyacente en los sintagmas verbales copulativos que, desde el punto de vista léxico, constituye el centro del predicado, pues su papel es llenar aquellos lexemas verbales de referencia vaga y asignarles un contenido concreto que limita su aplicación a la sustancia real. Parecida a ésta es la idea que sobre el predicativo sostiene $O$. Prytz, quien entiende por tal el adjetivo, sustantivo o infinitivo que, junto con el verbo, constituye el cuerpo central del predicado. $Y$ por completo idéntica a la de $E$. Alarcos es la concepción de M. L. Gutiérrez, que acepta el punto de vista de este autor y ve el atributo como el centro léxico del predicado en las oraciones copulativas ${ }^{3}$. Finalmente, podríamos afirmar que S. Gutiérrez Ordóñez define implícitamente el atributo como uno de los funtivos de la relación atributiva, relacionado con el tema de la atribución y con el verbo, definición que no guarda parentesco de ninguna clase con los tres tipos que acabamos de deslindar ${ }^{4}$.

1.1. Exégesis crítica. Si reflexionamos sobre las definiciones apuntadas, observamos que unas - las que se basan en la noción de cualidad- presentan los mismos inconvenientes que la caracterización de la atribución por este mismo procedimiento implica, básicamente que no siempre el atributo expresa una cualidad ${ }^{5}$; que otras - las que insisten en la peculiaridad del atributo de unirse al sujeto mediante un verbo- son circulares, pues, siguiendo en esto a J. Falk y a S. Gutiérrez Ordóñez, se define el atributo como palabra introducida por un verbo y éste como verbo que se construye con un atributo ${ }^{6}$. Tampoco parecen adecuadas las definiciones del atributo a partir de su oposición respecto a la cópula, por carecer ésta de significado léxico frente a aquél, pues la realidad de la lengua no responde a

3 Véase, respectivamente, J. Falk (1979), p. 19; Hernández (1970), pp. 67-68; C. Hernández (1984), pp. 149-151; E. Alarcos (1978), pp. 120-123; E. Alarcos (1985), pp. 15-16; O. Prytz (1979), pp. 86-87, y M. L. Gutiérrez (1978), pp. 55-56.

4 Véase S. Gutiérrez Ordóñez (1986), pp. 25-27.

5 Independientemente de las inadecuaciones a que conduce el definir los conceptos metalingüísticos mediante nociones tomadas de la filosofía y de la lógica aristotélicas (véase, al respecto, E. Coseriu (1972)), no siempre el atributo asigna una cualidad al sujeto y ello por expresiones del tipo «Madrid es la capital de España".

6 Vease, respectivamente, J. Falk (1979), p. 15 y S. Gutiérrez Ordóñez (1986), p. 22. El procedimiento recuerda, por otra parte, al utilizado tradicionalmente para definir el sujeto y el predicado de la oración: sujeto como aquello de lo que se predica algo y predicado como aquello que se predica del sujeto. Una revisión crítica de estos términos puede encontrarse en V. Báez San José (en prensa, b). 
tales planteamientos ${ }^{7}$. Finalmente, la que podríamos entender como definición de S. Gutiérrez Ordóñez es, más bien, una identificación del elemento que funciona como atributo, dado que lo que se nos dice de él es que está relacionado con el tema de la atribución y con el verbo.

De ahí que un primer punto que queda pendiente sea el de la definición del concepto de atributo. Nosotros, por considerar que las oraciones con el elemento llamado atributo constituyen esquemas oracionales ${ }^{8}$ que habrán de diferenciarse de otros esquemas oracionales del español mediante oposiciones privativas, graduales y equipolentes, creemos, en principio, que el atributo podría ser el determinador 1 del conjunto determinado constituido por el determinando 0 , sujeto del esquema oracional, más el núcleo predicativo ${ }^{9}$.

La dificultad de la definición propuesta sería su coincidencia con la proporcionada para el llamado complemento directo, pero aquélla quedaría obviada por la distinta naturaleza de la variable intralingüística que cumple la función de atributo, de algún modo en el caso de las oraciones atributivas y alguien o algo en el de las transitivas ${ }^{10}$, además de ser diferentes también las unidades que en la expresión textual invisten léxicamente tales variables y las proformas que sustituyen a aquéIlas ${ }^{11}$. A esto podría objetarse que la variable intralingüistica algo puede aparecer

7 Piénsese que, como muy bien afirma S. Gutiérrez Ordóñez (véase (1986), pp. 31-32), si los denominados copulativos no fueran unidades significativas, no existiria la diferencia de sentido que se da en las expresiones «Es triste»/«Está triste»/«Parece triste»/«Anda triste», etc.

8 Tomamos el término esquema oracional en el sentido que le da al mismo v. Báez San José (en prensa, a). En dicho artículo se establece una distinción entre a) evento de habla o expresión individual concreta; b) esquema oracional o signo ingüistico estático, considerado por abstracción como fuera del discurso conectado (texto) y fuera de la situación comunicativa, unidad que implica al menos un núcleo predicativo y, en casi todos los casos, una serie de variables intralingüisticas necesarias; asi el esquema oracional correspondiente a la expresión «Juan colocó el jarrón/al niño sobre la mesa» sería alguien coloca algo/a alguien en/sobre/... algún lugar, y c) expresión, elemento que representa todo el conjunto de adiciones significativas y variaciones que el esquema oracional estático adquiere al incluirse dentro de los varios dinamismos comunicativos lineales de tipo distinto: emisor, receptor, unidades léxicas que rellenan las variables intralingüísticas, esquema prosódico, etc.

9 En $\mathrm{V}$. Báez San José (en prensa b), después de analizar la relación de determinación expuesta por $\mathrm{N}$. Trubetzkoy (1939), "Le rapport entre le déterminé, le déterminant et le défini", Mélanges de Linguistique offerts à Ch. Bally sous les auspices de la Faculté de Lettres de l'Université de Genève, pp. 75-82, se apunta una concepción del núcleo predicativo de un esquema oracional como un modo del existir que puede ser determinado $\circ$ no, directa $\circ$ indirectamente, por variables intralingüisticas. Pensamos que a partir de ahí queda abierta la posibilidad de considerar el denominado atributo como una variable intralingüística determinadora del conjunto determinado formado por el Ilamado sujeto más el núcleo predicativo: $(\{$ lguien =determinando 0$)-$ núcleo predicativo =determinador 0$\}=$ determinado $1-$ de algún modo $=$ determinador 1, que en el nivel de la expresión podría investirse léxicamente como ((«Juan) es) muy bueno".

10 Por variable intralingüistica, elemento eventual de un determinado esquema oracional, entendemos la generalización absoluta no pronominal de los significados léxicos de las lenguas. Por el momento las variables intralingüisticas establecidas para el español son alguien, algo, algún momento, algún lugar y algún modo, correspondientes a los exofóricos quién, qué, cuándo, dónde y cómo (véase V. Báez San José (en prensa, a)). En este sentido, a expresiones atributivas como "El libro es rojo", por ejemplo, correspondería el esquema oracional algo es de algún modo, frente a expresiones transitivas como «Juan compró un esclavo" o "María compró el pescado en el supermercado", cuyo esquema oracional sería alguien compra algo.

11 Piénsese que, mientras la variable intralingüística de algún modo de un esquema oracional atributivo es investida en el dinamismo comunicativo de la expresión por las clases de palabras con significado categorial adjetivo y adverbio o por grupos de palabras formados por sustantivos introducidos por una 
como determinador 1 de esquemas oracionales tanto atributivos ${ }^{12}$ como transitivos, por lo que, en este caso, habría que estudiar la posibilidad de un sincretismo, consistente en que la variable a/go, variable estática en el esquema atributivo y en el no atributivo en sentido amplio, queda desambiguada en las expresiones textuales por distintas formas de pronominalización.

2. Caracterización formal. Además de definir el atributo, los gramáticos observan las características formales que los elementos que cumplen esta función poseen $y$, en este sentido, prácticamente todos ellos coinciden en afirmar que, al menos cuando el atributo es un adjetivo con variación genérica, concuerda en género y número con el sujeto de la atributiva y que, en caso de elisión, la forma pronominal átona lo actúa como sustituto, con la peculiaridad de que, por ser neutra, permanece invariable, aunque el elemento atributo elidido sea plural y femenino, por ejemplo, «Las paredes son altas» - «Las paredes lo son».

El hecho ha llamado la atención de los gramáticos, que han tratado por ello de buscarle explicaciones. $\mathrm{Y}$, así, A. Bello ${ }^{13}$ consideró que el 10 representativo de predicados era el caso complementario acusativo de ello, con lo que el verbo ser, que significa ser o estado pero no acción, se ha completado con un objeto paciente. El motivo, según A. Bello, hay que buscarlo en la incorporación de la fantasía al lenguaje ${ }^{14}$, de modo que en la existencia misma se puede fingir una especie de actividad. Para C. Hernández ${ }^{15}$, en cambio, los rasgos de género y número del atributo no necesitan reproducirse en la conmutación por el pronombre átono, ya que van marcados en el propio sujeto, de modo que por ser innecesarios y no comunes a todos los atributos no son precisos en la forma átona, que tiene, consecuentemente, un valor genérico neutro. Por el contrario, F. Carrasco ${ }^{16}$ propone una interpretación de esta proforma distinta por completo a las ofrecidas generalmente, pues, según este autor, lo no es el referente del sustantivo o del adjetivo atributo, sino del predicado nominal, entendiendo por tal la cópula más el atributo. La propia inaceptabilidad de variación en género y número demuestra, según él, que la referencia de la forma pronominal es la relación cópula más atributo, pues al no poseer este grupo predicativo ni género ni número lo apropiado es utilizar un referente

preposición (en otro apartado trataremos de las proformas que sustituyen a tales unidades), las variables intralingüisticas alguien y algo de esquemas oracionales transitivos son investidas léxicamente por unidades de la clase sustantivo o por oraciones subordinadas sustantivas, de acuerdo con la denominación de las gramáticas tradicionales, cuando la variable es algo; respecto a las proformas que las sustituyen, éstas son lollos, lallas y lelles.

12 En el caso de expresiones como «El tabaco es un peligro para la salud» el esquema oracional correspondiente, algo es algo, presenta la variable intralingüística algo tanto en la función llamada sujeto, determinando 0 del esquema oracional, según nuestra concepción, como en la denominada atributo, determinador 1 del conjunto determinado constituido por el determinado 0 más el núcleo predicativo, de acuerdo con nuestros presupuestos teóricos.

13 Véase A. Bello (1984), pp. 120-121.

14 Es posible que esta afirmación del gramático venezolano tenga que ver con alguna influencia del romanticismo y de G. Vico en cuanto al concepto de universal fantástico — no olvidemos que A. Bello fue un excelente traductor de obras científicas y literarias italianas-.

15 Véase C. Hernández (1984), pp. 73-74.

16 Véase F. Carrasco (1972). (1973) y (1975). 
neutro respecto a tales características morfológicas. Por otra parte, el hecho de que lo no aparezca como proforma de los verbos utilizados como copulativos induce también a F. Carrasco a considerarlo sustituto del predicado nominal entero, pues en construcciones como "Llegó cansado" o «Andaba triste" lo que existe, para este autor, es un auténtico predicado verbal y no uno nominal, de ahí la imposibilidad de pronominalización con lo y la referencia de éste a ser o estar más el atributo ${ }^{17}$. Hasta tal punto los gramáticos han dado importancia a esta característica formal que comentamos que para E. Alarcos, por ejemplo, el rasgo esencial de la función atributo es la posibilidad de ser sustituido por la invarable 10 , incremento del núcleo verbal, pues el rasgo de la concordancia en género y número no siempre se cumple. De manera análoga, para $\mathrm{R}$. Cano Aguilar la reproducción del elemento adyacente a ser, estar y parecer por el pronombre invariable lo es el fundamento de la distinción entre estructuras predicativas y estructuras atributivas ${ }^{18}$.

2.1. Las proformas del atributo como criterio clasificador de los núcleos predicativos de las oraciones atributivas. La cuestión del referente pronominal del atributo nos parece que debe relacionarse con el tipo de unidad que inviste léxicamente la variable intralingüística que funciona como atributo. En efecto, si, frente a ser, estar y parecer, otros copulativos no aceptan la proforma átona y neutra lo, es porque tampoco registran como atributo una unidad de la clase de los sustantivos y sí, en cambio, un adjetivo calificativo, un adverbio o un sustantivo precedido por una preposición, de modo que en este caso tenemos como sustituto pronominal las proformas asi o tal. Además de que ser, estar y parecer, por poder llevar como atributo un adjetivo, un adverbio y un sintagma preposicional, admiten también estas mismas proformas.

Queremos decir con todo ello que para cada verbo atributivo ${ }^{19}$ habría que especificar todas las posibiidades de realización en el discurso de la variable de algún modo y de la variable algo, para pasar después a comprobar cuáles son las proformas que sustituyen a cada una de las posibilidades en cuestión y para, asi, ver si es posible establecer oposiciones entre los núcleos predicativos de la clase que, en un primer momento, podemos llamar atributiva. Téngase en cuenta, por ejemplo, que verbos como elegir, nombrar, proclamar, etc., exigen como atributo la variable intralingüística algo, investida léxicamente sólo mediante sustantivos que se sustituyen únicamente por proformas como esto, eso o aquello. A este punto de la investi-

17 No obstante, este autor no parece tener en cuenta que el verbo parecer acepta la proforma lo: "Parecen hermosas" - "Lo parecen", aunque su interpretación de esta estructura superficial como derivada de la profunda "Parece que son hermosas" - «Parece que lo son" - «Lo parecen», le resuelva, sólo aparentemente, el problema.

18 Véase, respectivamente, E. Alarcos (1985), pp. 15-16 y R. Cano Aguilar (1983), pp. 9-11

19 No consideramos como verbos de las expresiones atributivas sólo los tradicionales copulativos, ser y estar, sino que, en principio, atribuimos tal carácter a todos los verbos del español que en un esquema oracional se constituyen en núcleos predicativos que exigen, obligatoriamente, la presencia de la variable intralingüística de algún modo, al menos como determinador 1 del esquema oracional (en el tercer apartado trataremos la posibilidad del atributo como determinador 2), o de la variable algo, teniendo en cuenta, en este caso, que en el dinamismo de la expresión las proformas que la sustituyen posibilitan su distinción respecto a la variable algo de un esquema transitivo. 
gación empírica que estamos proponiendo ${ }^{20}$ podría objetarse que estar no admite atributos sustantivos y, sin embargo, no rechaza como proforma el neutro átono 10. El fenómeno podría explicarse, tal vez, diacrónicamente y, en este sentido, cabría reflexionar sobre si la posibilidad de la proforma lo con estar, a pesar de que no sustituye a una unidad de la clase de los sustantivos, no estaría en relación con el incremento de valores expresados con estar a costa del copulativo ser ${ }^{21}$.

3. Unidades que cumplen la función de atributo. En lo que se refiere a la clase de unidades que pueden cumplir tal función, los gramáticos del español ${ }^{22}$ enumeran las distintas posibilidades de acuerdo con el verbo que aparece con el atributo. Ser, por ejemplo, puede ir acompañado de atributos sustantivos, incluso formando un sintagma preposicional; adjetivos, incluidos determinativos y calificativos; pronominales, $y$, en este caso, es posible encontrar pronombres personales, interrogativos, demostrativos, posesivos, numerales y ordinales; sus atributos pueden ser también infinitivos; participios adjetivados; adverbios, y oraciones sustantivadas. Por su parte, la relación de elementos atributos de estar es más reducida, pues este copulativo sólo puede enlazar con el sujeto sintagmas preposicionales, adjetivos calificativos y adverbios. S. Gutiérrez Ordóñez llega a considerar como atributos de verbos distintos a ser, estar y parecer construcciones absolutas como el sintagma preposicional de "Van pálidos, con la cara sucia»; sintagmas de valor consecutivo: «En primavera se pone que no hay quien le trate»; sintagmas preposicionales del tipo «Dan en temerarios», y gerundios, aunque, en este caso, como atributos de implemento: «Imagino a Rodin diseñando sus esculturas» ${ }^{23}$.

3.1. Exégesis crítica. Los problemas que en este apartado se plantean se refieren a los participios, gerundios e infinitivos como atributos, que encontramos ejemplificados en las siguientes expresiones proporcionadas por J. Martínez Álvarez ${ }^{24}$ : "El texto está refundido", "El niño estaba durmiendo» y «Oigo subir el ascensor». Por lo que respecta al infinitivo como atributo de implemento, el acuerdo entre los

20 Este artículo puede entenderse, de algún modo, como fundamento teórico de una investigación empirica que estamos llevando a cabo sobre los núcleos predicativos atributivos del español y que se inserta en el proyecto dirigido por V. Báez San José, "Esquemas sintáctico-semánticos del español». La finalidad del mismo es establecer los esquemas sintáctico-semánticos correspondientes a todos los núcleos predicativos del español y, en este sentido, nuestro ámbito de estudio está restringido, por el momento, a los que, por ahora, estamos llamando núcleos predicativos atributivos, para llegar al establecimiento de sus correspondientes esquemas sintáctico-semánticos y a la determinación de las oposiciones funcionales que entre los mismos existan.

21 Desde un punto de vista típológico, esta precisión diacrónica podría estar avalada por la comparación con el italiano, donde tenemos essere y stare, pero, al encontrarse más cerca la lengua italiana de la latina, el verbo stare no ha desarrollado los esquemas oracionales del estar castellano. Otras lenguas, como el catalán, por ejemplo, representan, a su vez, estadios intermedios entre la situación del castellano y del italiano (véase, en este sentido, J. Falk (1979), pp. 7-8 y 145-146).

22 Véase, por ejemplo, R.A.E. (1931), pp. 158-159; R.A.E. (1977), pp. 364-367; F. Carrasco (1974); M. L. Gutiérrez (1978), pp. 192-197, y C. Hernández (1984), p. 151.

23 Véase S. Gutiérrez Ordóñez (1986), pp. 113-136.

24 Véase J. Martínez Álvarez (1985), pp. 116-117. 
gramáticos no es unánime y ya desde que $E$. Alarcos identificó esta función del infinitivo ${ }^{25}$ se han expuesto diversas objeciones a la misma. Asi, por ejemplo, J. A. de Molina Redondo ${ }^{26}$ opina que "Veía florecer los claveles" y "Veía regar los claveles" son estructuras idénticas, frente a la postura de aquel autor, que en la primera expresión identifica florecer como atributo del implemento los claveles, mientras que en la segunda el infinitivo, con su implemento los claveles, sería, a su vez, implemento de veía.

Sin entrar, por ahora, a fondo en la polémica ${ }^{27}$, pensamos que no se pueden considerar estas formas atributos sin demostrar, previamente, que el participio y el gerundio no son núcleos predicativos determinados por un auxiliar o un semiauxiliar. Es decir, a priori, no se puede desechar la posibilidad de que los considerados verbos copulativos de «está refundido» y «estaba durmiendo» aporten una determinación, al menos aspectual, del que sería núcleo predicativo en un esquema oracional: refundido y durmiendo ${ }^{28}$. En cuanto al infinitivo atributo en oraciones no identificativas, habría que reflexionar sobre la equivalencia distribucional que mantiene con construcciones en las que el verbo aparece en forma personal: "Oigo subir el ascensor» - "Oigo que el ascensor sube», de ahi la posibilidad de considerar ambas construcciones como variantes discursivas libres de la variable intralingüística algo del esquema no atributivo alguien oye a alguien/algo.

4. Clasificación de la función atributo. Cuanto acabamos de señalar se refiere, básicamente, al atributo de las oraciones con los verbos ser y estar, pero, dado que los gramáticos han llegado a diferenciar distintos tipos para la función que comentamos, vamos a dedicar un último apartado al estudio de las clases establecidas, aunque, para no repetir ideas y conceptos con la exposición de las diferentes tipologías, nos limitaremos a presentar una única clasificación, donde queden recogidas, en la medida de lo posible, las variantes señaladas por distintos autores.

a) Un primer tipo de atributo correspondería al que ya tenemos caracterizado, más al atributo de otros verbos considerados copulativos como, por ejemplo, parecer, quedar, resultar o salir. Sin embargo, los gramáticos que estudian pormenorizadamente esta función - O. Prytz, B. Rodríguez Díez o S. Gutiérrez Ordóñez ${ }^{29}$

25 Véase E. Alarcos (1978), pp. 133-142 y 296-313.

26 Véase J. A. de Molina (1971).

27 Con todo, importa subrayar que las razones de E. Alarcos para asignar al infinitivo de «Oigo sonar las campanas" y "Veia florecer los claveles" la función de atributo de implemento no parecen adecuadas. pues de acuerdo con su razonamiento: «En /6/ Oigo sonar las campanas, es posible decir las oigo sonar, pero no Oigo sonarlas. Luego /las campanas/ es implemento y/sonar/su atributo" (véase E. Alarcos (1978), pp. 296-313), lo que puede concluirse, evidentemente, es que las campanas no es implemento de sonar, no que sonar sea atributo de las campanas.

28 Tanto más cuanto que un siglo de investigación diacrónica nos ha demostrado que todas las formas sintéticas del verbo en estadios anteriores han sido formas analíticas y que, además, E. Coseriu en un excelente trabajo sobre el aspecto (véase E. Coseriu (1980)), aplicado al español por $\mathrm{N}$. Cartagena (véase $\mathrm{N}$. Cartagena, 1978)), ha hablado de múltiples perífrasis aspectuales, todas analíticas y donde el núcleo predicativo es el infinitivo, gerundio o participio: ando fastidiado, va a comenzar, vengo estudiando, etc.

29 Véase, respectivamente, O. Prytz (1979), pp. 79-80; B. Rodríguez Diez (1982), pp. 36-37, y S. Gutiérrez Ordóñez (1986), pp. 31-40. 
separan en esta clase uno del otro en razón del referente pronominal que los sustituye: lo en el caso de ser, estar y parecer y asi y también tal en el caso de los otros copulativos, aunque ambos coincidan en las formas interrogativas con las que se pregunta por ellos: ¿qué? y ¿cómo?

b) El segundo tipo al que queremos referirnos es el denominado atributo de sujeto, predicativo adverbial o aditamento atributivo, por la calificación modal que con él se realiza también sobre el verbo. «El niño duerme tranquilo» o «Los sabios pasean meditabundos» serían algunas de las expresiones aducidas para ejemplificar esta clase de atributo, que, frente al anterior, nunca podria ser sustituido por la forma pronominal 10 , sino que tendríamos que referirnos a él, en un contexto en el que se da por sabido, con la forma asi. Desde la propuesta de E. Alarcos ${ }^{30}$, estas estructuras predicativas que incluyen una relación de atribución suelen interpretarse como híbridas, así «El niño viene cansado", por ejemplo, se considera resultado de «El niño viene» más «El niño está - parece cansado», a partir de la supresión de los elementos léxicos comunes ${ }^{31}$.

c) Otra clase de atributo corresponde al denominado predicativo o atributo de implemento, de acuerdo con la denominación funcional del complemento directo. Expresiones como las de «Llevaba rotos los zapatos» o «Eligieron a Juan presidente" responderían a este tercer tipo, cuyos referentes pronominales son asi, cuando se trata de un atributo adjetivo, y ello, esto, eso o aquello cuando el atributo es una unidad de la clase de los sustantivos. Posee las mismas características que el de sujeto, con la particularidad de que, en este caso, el tema de la atribución es el complemento directo.

d) S. Gutiérrez Ordóñez ${ }^{32}$ habla también de un atributo para la función oracional de suplemento y así habría que entender los sintagmas introducidos por como en las expresiones «Hablan de Carrillo como próximo embajador de un país del Este» o «Pasan de ti como catedrático, pero no como persona».

e) Un quinto tipo seria el de complemento ${ }^{33}$, siguiendo de nuevo la terminología de $\mathrm{E}$. Alarcos, atributo que B. Rodríguez Díez observa en el adjetivo de «Al vendedor los ladrones le arrebataron las jcyas inconsciente», S. Gutiérrez Ordóñez, en el sustantivo asno de "Si al hombre que es bueno debieran decirle asno» y J. Martínez Álvarez, en el adjetivo de «María se puso el abrigo rápida», teniendo en cuenta que el se funciona como complemento ${ }^{34}$.

30 Véase E. Alarcos (1978), pp. 120-123.

31 Sin embargo, nada hay en la expresión “El niño viene cansado» que implique tal estructura hibrida. Sólo a nivel conceptual, claro está, si un hablante dice de alguien que viene cansado, es porque a él le parece cansado o le parece que está cansado. Por otra parte, esta interpretación recuerda el método de la gramática generativa, a pesar de la perspectiva funcional de quienes la sustentan, aunque nada obstaria a ello, si el procedimiento utilizado hubiera sido explicado de manera previa y razonada.

32 Véase, por ejemplo, S. Gutiérrez Ordóñez (1986), p. 148.

33 Véase, respectivamente, B. Rodríguez Diez (1982), pp. 42-44; S. Gutiérrez Ordóñez (1986), pp. 134135, y J. Martínez Álvarez (1985), p. 112.

34 No obstante, este grupo resulta problemático, al menos por las ejemplificaciones aducidas. En efecto, inconsciente en «Al vendedor los ladrones le arrebataron las joyas inconsciente», puede, por su movilidad, interpretarse también como un adyacente del nombre o como un atributo incidental, caracterizado, entre otras particularidades, de acuerdo con S. Gutiérrez Ordóñez (véase (1986), pp. 143-152), por ir entre pausas y por un leve descenso en la frecuencia de tono fundamental. Por su parte, nada parece impedir que el tema del atributo asno en "Si al hombre que es bueno debieran decirle asno» funcione como 
f) B. Rodríguez Díez establece un nuevo tipo para lo que él llama atributo del adyacente temático y, pensando que por adyacente temático se entiende el sujeto léxico de una forma nominal del verbo, este atributo quedaría ejemplificado en el adjetivo de "Viviendo satisfechos evitamos enfermedades" 35 .

g) Tanto el autor anterior como S. Gutiérrez Ordóñez mencionan la existencia de unos llamados atributos preposicionales, que unas veces lo son de sujeto: «Dan en temerarios", y otras de implemento: "Lo tenían por loco» ${ }^{36}$.

h) Un último tipo podría corresponder al denominado atributo en cláusulas absolutas, ejemplificado en el primer sintagma de clniciada la carrera se destaca el jamaicano". Las peculiares características de estas construcciones hacen que no nos detengamos ahora en ellas; con todo, puede verse el tratamiento bastante pormenorizado que de las mismas ofrece S. Gutiérrez Ordóñez ${ }^{37}$.

4.1. Exégesis crítica. La lectura del apartado anterior habrá mostrado, muy probablemente, que las clasificaciones recogidas entremezclan criterios como el tipo de verbo de la oración, la función del tema del atributo y las características formales del atributo (atributos preposicionales/atributos no preposicionales). Frente a tal disparidad, nos parece que, de establecerse una clasificación, debería partirse de a obligatoriedad de este elemento en un esquema oracional con un núcleo predicativo concreto.

Desde ahí habria que especificar el carácter biactancial o triactancial del esquema en cuestión y, si en el caso de un esquema biactancial como el correspondiente a «Juan continúa enfermo", tendríamos un núcleo predicativo, continúa, determinador 0 del determinando 0 , sujeto de la oración, Juan, y un atributo, enfermo, determinador 1 de la relación ya instaurada entre el núcleo predicativo y el sujeto, Juan continúa, en un esquema triactancial, por ejemplo el que subyace a «Los ciudadanos eligieron a Juan presidente», estaríamos ante la siguiente serie de relaciones de determinación: el determinador 0 , eligieron, núcleo predicativo del esquema, determina directamente al sujeto del mismo, los ciudadanos, el conjunto se constituye, a su vez, en un determinando sobre el que incide el determinador 1 , a Juan, y el nuevo conjunto es determinado, por. último, por el determinador 2, presidente.

Creemos que, de este modo, se eliminarian, en la clasificación de los tipos de

implemento en vez de complemento, con lo que en esa expresión no se daría esta clase de atributo. Por último, si en la expresión «Maria se puso el abrigo rápida» sustituimos la forma se por otra no correferencial con el llamado sujeto, "María le puso el abrigo rápida", se hablaría de un atributo de sujeto y no de complemento, por lo que no se ve clara la razón para no seguir considerando rápida atributo de sujeto en la primera expresión.

35 Véase B. Rodríguez Díez (1982), pp. 44-45. Ahora bien, si adyacente temático es el sujeto léxico de una forma nominal del verbo, esta clase de atributo parece innecesaria al haber fijado ya un atributo de sujeto, aunque el verbo estuviera en forma personal: «Mis padres vivieron felices".

36 Véase B. Rodríguez Díez (1982), pp. 45-46 y S. Gutiérrez Ordóñez (1986), pp. 135-143. El tipo en cuestión parece establecido más por un criterio formal —un elemento introducido por una preposición es el atributo-, que en virtud de la función desempeñada por el tema del atributo, pues, según el tema del atributo, se habla, posteriormente, como se ha visto, de grupos ya establecidos: atributos de sujeto: «Dan en temerarios", y atributos de implemento: "Lo tenian por loco".

37 Véase S. Gutiérrez Ordóñez (1978) y (1986), pp. 153-199. 
atributo, las incoherencias anteriormente señaladas, además de que se podría distinguir entre una función atributo en el nivel abstracto oracional, obligatoria para la constitución de determinados esquemas sintáctico-semánticos, y una más de las determinaciones del sintagma nominal en el nivel de la expresión; compárese, por ejemplo, "Juan lleva zapatos", "Juan lleva un zapato», "Juan lleva los zapatos", «Juan lleva los zapatos rojos» y «Juan lleva rotos los zapatos», expresión esta última que ha inducido a los gramáticos a hablar de un atributo de implemento, cuando el esquema oracional de todas las expresiones anteriores no atributivas es alguien lleva a alguien/algo.

5. Como conclusión queremos señalar que el estatus problemático del concepto atributo exige continuar el estudio de aquellas expresiones en que se muestra este fenómeno. De ahí que, en la investigación a que más arriba hemos aludido, hayamos empezado por recoger los verbos que en español pueden constituirse en núcleos predicativos de oraciones atributivas. Así, con la observación de un corpus que recoja, ampliamente, construcciones con estos verbos y desde los presupuestos teóricos y metodológicos que anteriormente hemos tan sólo insinuado, creemos que puede llegarse a un análisis más coherente y adecuado de la noción de atributo, con la finalidad de proporcionar una definición y clasificación de esta función oracional más ajustadas a la realidad de la lengua.

\section{Bibliografía}

Alarcos Llorach, E. (1978, 2. ${ }^{a}$ ed.), «Verbo transitivo, verbo intransitivo y estructura del predicado", Estudios de gramática funcional del español, Madrid, pp. 109-123.

- $\left(1978,2^{2}{ }^{2}\right.$ ed.), “Algunas construcciones del infinitivo», Estudios de gramática funcional del español, Madrid, pp. 133-142.

- $\left(1978,2 .^{a}\right.$ ed.), «Términos adyacentes del infinitivo», Estudios de gramática funcional del español, Madrid, pp. 296-313.

- (1985), "Otra vez sobre pasividad y atribución en español», Lecciones del I y II Curso de Lingüistica Funcional (1983 y 1984), Oviedo, pp. 15-21.

Alcina, J. y J. M. Blecua (1975), Gramática española, Barcelona.

Báez San José, V. (en prensa, a), “Oración y esquema oracional», Homenaje al Profesor D. Julio Fernández Sevilla.

- (en prensa, b), «Determinación, predicación y el problema del sujeto», Homenaje al Profesor D. Félix Monge.

Bello, A. (1984), Gramática de la lengua castellana, Madrid.

Cano Aguilar, R. (1983), El predicado verbal, Madrid.

Carrasco, F. (1972), «El pronombre neutro lo como pro-forma del predicado nominal", Boletín del Instituto Caro y Cuervo, XXVII, pp. 324-333.

- (1973), "Nota adicional a 'El pronombre neutro lo como pro-forma del predicado nominal'», Boletín del Instituto Caro y Cuervo, XXVIII, pp. 108-111.

- (1974), "Ser/ v /Estar y sus repercusiones en el sistema", Boletín del Instituto Caro y Cuervo, XXIX, 2, pp. 316-349. 
- (1975), «Remarques sur le comportement des clitiques neutres dans le système attribut de l'espagnol et du français", Revue Romane, pp. 293-305.

Cartagena, N. (1978), «Acerca de las categorías de tiempo y aspecto en el sistema verbal del español», Revista Española de Lingüística, 8, 2, pp. 373-408.

Coseriu, E. (1972), "Sobre las categorías verbales («Partes de la oración»)", Revista de Lingüística Teórica y Aplicada, 10, pp. 7-25.

- (1980), «La notion d'aspect», en J. David y R. Martin (eds.), Colloque organisé par le Centre d'Analyse Syntaxique de l'Université de Metz (18-20 mai 1978), Études publiées par le Centre d'Analyse Syntaxique de I'Université de Metz, pp. $13-25$.

Falk, J. (1979), Ser y Estar con atributos adjetivales. Anotaciones sobre empleo de la cópula en catalán y en castellano, 1, Uppsala.

Gili Gaya, S. (1976, 11. a ed.), Curso superior de sintaxis española, Barcelona.

Gutiérrez, M. L. (1978), Estructuras sintácticas del español actual, Madrid.

Gutiérrez Ordóñez, S. (1978), "Construcciones atributivas absolutas», Verba, 5, pp. 305-314.

- (1986), Variacioens sobre la atribución, León.

Hernández, C. (1970), Sintaxis española, Valladolid.

- (1984), Gramática funcional del español, Madrid.

Marcos Marín, F. (1975, 3. ${ }^{a}$ ed.), Aproximación a la gramática española, Madrid.

Martínez Álvarez, J. (1985), "Sobre algunas estructuras atributivas", Lecciones del I y // Curso de Lingüística Funcional (1983 y 1984), Oviedo, pp. 111-119.

Molina, J. A. de (1971), "La construcción 'verbo en forma personal+infinitivo'», Revista Española de Lingüística, 1, 2, pp. 275-298.

Navas Ruiz, R. (1963), Ser, y estar. Estudio sobre el sistema atributivo del español, Salamanca.

Prytz, O. (1979), "Situación del predicativo español», Mélanges d'études romanes offerts à Leiv Flydal, Etudes Romanes de l'Université de Copenhague, Revue Romane, numero spécial 18, Akademik Forlag, pp. 78-88.

Real Academia Española (nueva edición, reformada, de 1931), Gramática de la Lengua Española, Madrid, (1959), E. Espasa-Calpe.

- (1977, 4. ${ }^{a}$ reimp.), Esbozo de una nueva gramática de la lengua española, Madrid.

Rogríguez Díez, B. (1982), «L'attribut en espagnol: essai d'une description et classification fonctionnelles", La Linguistique, vol. 18, fasc. 2, pp. 33-48.

Seco, R. (1971, 9. ${ }^{a}$ ed., 3. ${ }^{2}$ reimp.), Manual de gramática española, Madrid. 